\title{
SuperHERO: the next generation hard x-ray HEROES telescope
}

Author(s): Jessica A. Gaskin, NASA Marshall Space Flight Ctr.; Steven D. Christe, NASA Goddard Space Flight Ctr.; Colleen Wilson-Hodge, NASA Marshall Space Flight Ctr.; Albert Y.

M. Shih, NASA Goddard Space Flight Ctr.; Brian D. Ramsey, Allyn F. Tennant, , NASA Marshall Space Flight Ctr., Douglas A. Swartz, USRA

SuperHERO is a new high-sensitivity Long Duration Balloon (LDB)-capable, hard-x-ray (20-75 $\mathrm{keV}$ ) telescope for making novel astrophysics and heliophysics observations. The proposed SuperHERO payload will be developed jointly by the Astrophysics Office at NASA Marshall Space Flight Center, the Solar Physics Laboratory and Wallops Flight Facility at NASA Goddard Space Flight Center. SuperHERO is a follow-on payload to the High Energy Replicated Optics to Explore the Sun (HEROES) balloon-borne telescope that recently launched from Fort Sumner, NM in September of 2013. The HEROES core instrument is a hard x-ray telescope consisting of x-ray 109 optics configured into 8 modules. Each module is aligned to a matching gas-filled detector at a focal length of $6 \mathrm{~m}$. SuperHERO will make significant improvements to the HEROES payload, including: new solid-state multi-pixel CdTe detectors, additional optics, the Wallops Arc-Second Pointer, alignment monitoring systems and lighter gondola. 